\title{
INTEGRAL OF AN E-FUNCTION EXPRESSED AS \\ A SUM OF TWO $E$-FUNCTIONS
}

\author{
by T. M. MACROBERT
}

(Received 21st August, 1951)

$\$ 1$. Introductory. The formula to be proved is

$$
\begin{aligned}
& \frac{1}{\Gamma\left(\rho_{q+1}-\alpha_{p+1}\right)} \int_{0}^{1} t^{-\rho_{q+1}}(1-t)^{\rho_{q+1}-\alpha_{p+1}-1} E\left(p ; \alpha_{r}: q ; \rho_{s}: x t\right) d t \\
& \quad=\frac{\sin \left(\alpha_{p+1} \pi\right)}{\sin \left(\rho_{q+1} \pi\right)} E\left(p+1 ; \alpha_{r}: q+1 ; \rho_{s}: x\right) \\
& +\frac{\sin \left(\alpha_{p+1}-\rho_{q+1}\right) \pi}{\sin \left(\rho_{q+1} \pi\right)} x^{\rho_{q+1}-1} E\left(p+1 ; \alpha_{r}-\rho_{q+1}+1: 2-\rho_{q+1}, \rho_{1}-\rho_{q+1}+1, \ldots, \rho_{q}-\rho_{q+1}+1: x\right),(1) \\
& \text { where } \rho_{q+1}-\alpha_{p+1}>0, \alpha_{r}-\rho_{q+1}+1>0, r=1,2, \ldots, p, \text { and } p \geqq q+1 .
\end{aligned}
$$

The following formulae are required in the proof:

where $p \geqq q+1$ and

$$
E\left(p ; \alpha_{r}: q ; \rho_{s}: x\right)=\sum_{r=1}^{p} P\left(\alpha_{r} ; p-1 ; \alpha_{s}: q ; \rho_{t}: x\right)
$$

$$
\begin{gathered}
P\left(\alpha_{r} ; p-1 ; \alpha_{s}: q ; \rho_{t}: x\right)=\frac{\prod_{s=1}^{p} \Gamma\left(\alpha_{s}-\alpha_{r}\right)}{\prod_{t=1}^{q} \Gamma\left(\rho_{t}-\alpha_{r}\right)} \Gamma\left(\alpha_{r}\right) x^{\alpha_{r}} \\
\times F\left\{\begin{array}{l}
\alpha_{r}, \alpha_{r}-\rho_{1}+1, \ldots, \alpha_{r}-\rho_{q}+1 ;(-1)^{p-q} x \\
\alpha_{r}-\alpha_{1}+1, \ldots * \ldots, \alpha_{r}-\alpha_{p}+1
\end{array}\right\},
\end{gathered}
$$

$r=1,2,3, \ldots, p$; when $p=q+1,|x|<1$ :

$$
\sin (\gamma \pi) \sin (p-\alpha) \pi+\sin (\gamma-\rho) \pi \sin (\alpha \pi)=\sin (\rho \pi) \sin (\gamma-\alpha) \pi \text {. }
$$

§ 2. Proof of the Formula. On applying (2) on the L.H.s. of (1), it becomes $\sum_{r=1}^{p} I_{r}$, where

$$
\begin{aligned}
& I_{r}=\frac{\Gamma\left(\alpha_{r}-\rho_{q+1}+1\right)}{\Gamma\left(\alpha_{r}-\alpha_{p+1}+1\right)} \frac{\prod_{8=1}^{p} \Gamma\left(\alpha_{s}-\alpha_{r}\right)}{\prod_{t=1}^{q} \Gamma\left(\rho_{t}-\alpha_{r}\right)} \Gamma\left(\alpha_{r}\right) x^{\alpha_{r}} \\
& \times F\left\{\begin{array}{l}
\left.\alpha_{r}, \alpha_{r}-\rho_{1}+1, \ldots, \alpha_{r}-\rho_{q+1}+1 ;(-1)^{p-q} x\right\} \\
\alpha_{r}-\alpha_{1}+1, \ldots * \ldots, \alpha_{r}-\alpha_{p+1}+1
\end{array}\right\}
\end{aligned}
$$

$r=1,2,3, \ldots, p$.

Now the R.H.s. of ( 1 ) is equal to

$$
\begin{aligned}
& \sum_{r=1}^{p}\left[\frac{\sin }{\sin } \frac{\left(\alpha_{p+1} \pi\right)}{\left(\rho_{q+1} \pi\right)} \frac{\sin }{\sin } \frac{\left(\rho_{q+1}-\alpha_{r}\right) \pi}{\left(\alpha_{p+1}-\alpha_{r}\right) \pi}+\frac{\sin \left(\alpha_{p+1}-\rho_{q+1}\right) \pi}{\sin \left(\rho_{q+1} \pi\right)} \frac{\sin \left(\alpha_{r} \pi\right)}{\sin \left(\alpha_{p+1}-\alpha_{r}\right) \pi}\right] I_{r} \\
& +\left[\frac{\sin \left(\alpha_{p+1} \pi\right)}{\sin \left(\rho_{q+1} \pi\right)}+\frac{\sin \left(\alpha_{p+1}-\rho_{q+1}\right) \pi}{\sin \left(\rho_{q+1} \pi\right)} \frac{\sin \left(\alpha_{p+1} \pi\right)}{\sin \left(\rho_{q+1}-\alpha_{p+1}\right) \pi}\right] P\left(\alpha_{p+1} ; p ; \alpha_{s}: q+1 ; \rho_{t}: x\right) \text {. }
\end{aligned}
$$

Here the last term is zero, and the sum of the remaining terms, by (4), is $\sum_{r=1}^{p} I_{r}$. Thus the formula has been proved.

University OF GLasgow 